\title{
Lumbar Intradural Neurocysticercosis: A Case Report
}

\author{
Sang-Beom Han, Hyon-Jo Kwon, Seung-Won Choi, Hyeon-Song Koh, \\ Seon-Hwan Kim, Shi-Hun Song, Jin-Young Youm \\ Department of Neurosurgery, Chungnam National University School of Medicine, Daejeon, Korea
}

Cysticercosis is the most common parasitic disease affecting the central nervous system. Spinal involvement is rare in neurocysticercosis, and isolated spinal involvement without evidence of cranial involvement is even rarer. We report an unusual case of neurocysticercosis with isolated spinal involvement. A 59 year-old male presented with radiating pain in the left leg. He complained of aggravating weakness and numbness in the left leg since his previous visit one month ago. Magnetic resonance imaging (MRI) revealed multiple peripheral wall-enhanced intradural cystic masses from L1 to L5. The patient underwent a total laminectomy of L4. Dissection revealed abnormal cystic masses compressing the nerve roots. The cyst was punctured, spilling clear mucoid fluid into the surgical field. The exposed cysticerci, white and mucoid, was easily removed. Patient received course of steroids and oral albendazole. The patient experienced symptomatic improvement without further neurologic deficits except for mild sensory impairment. Clinicians should include spinal neurocysticercosis in differential diagnosis of radiculopathies. Although isolated spinal neurocysticercosis is rare, it can be satisfactorily managed with surgery and medication.

Key Words: Neurocysticercosis $\cdot$ Cysticercosis $\cdot$ Spine $\cdot$ Intradural

\section{INTRODUCTION}

Cysticercosis, caused by the tapeworm Taenia Solium, is the most common parasitic disease affecting the central nervous system ${ }^{1}$. Cysticercosis typically infiltrates the brain parenchyme, while spine infection of neurocysticercosis is rare with incidence of $1-3 \%^{1,2,15)}$. In addition, the neurocysticercosis, not invading the brain, is even more rare because most cases of spinal neurocysticercosis accompanies brain intrusion ${ }^{1)}$. We report an unusual case of neurocysticercosis of isolated spinal involvement.

\section{CASE REPORT}

A 59-year-old male presented with 9 month long radiating

- Received: August 2, 2014 - Revised: August 25, 2014

- Accepted: September 1, 2014

Corresponding Author: Jin-Young Youm, MD

Department of Neurosurgery, School of Medicine, Chungnam National

University, 282 Munhwa-ro, Jung-gu, Daejeon 301-721, Korea

Tel: +82-42-280-7361, Fax: +82-42-280-7363

E-mail: jyyoum@cnu.ac.kr

అThis is an Open Access article distributed under the terms of the Creative

Commons Attribution Non-Commercial License (http://creativecommons.org/ licenses/by-nc/3.0/) which permits unrestricted non-commercial use, distribution, and reproduction in any medium, provided the original work is properly cited. pain in the left lower limb. He claimed the symptoms of weakness and numbness had aggravated, although without bladder symptoms, since his last visit 1 month ago. Manual muscle testing (MMT) revealed grade 4/5 in the muscle of the left lower limb and sensory perceptions in both lower limbs were decreased along bilateral L5 and S1 dermatomes. The Babinski sign was negative bilaterally. Neither particular travel experience nor history of eating raw food was reported by the subject. Eosinophil in the complete blood count was measured at $1.5 \%$, and showed no eosinophilia. Other blood counts and chemistry were in the normal range. An enzyme-linked immunosorbent assay (ELISA) test for anti-Taenia solium $\operatorname{IgG}$ in the serum was negative. Magnetic resonance imaging (MRI) revealed multiple peripheral wall-enhanced intradural cystic masses from L1 to L5. The largest lesion was a $1.4 \times 1.3 \times 3.0 \mathrm{~cm}$ cystic mass in L3 to L4. The lesion was well-circumscribed and located in the intradural extramedullary area. The MRI signal intensity of the lesion was similar to cerebrospinal fluid; low in T1 weighted image and high in T2 weighted image (Fig. 1). The brain MRI was normal.

Surgery was performed while utilizing nerve detecting devices monitoring the somatosensory evoked potential (SSEP), motor evoked potential (MEP) and electromyography (EMG) of the patient. The patient underwent a total laminectomy of L4 and a partial laminectomy of L3. The dura was opened in midline under an operating microscope, showing severe 

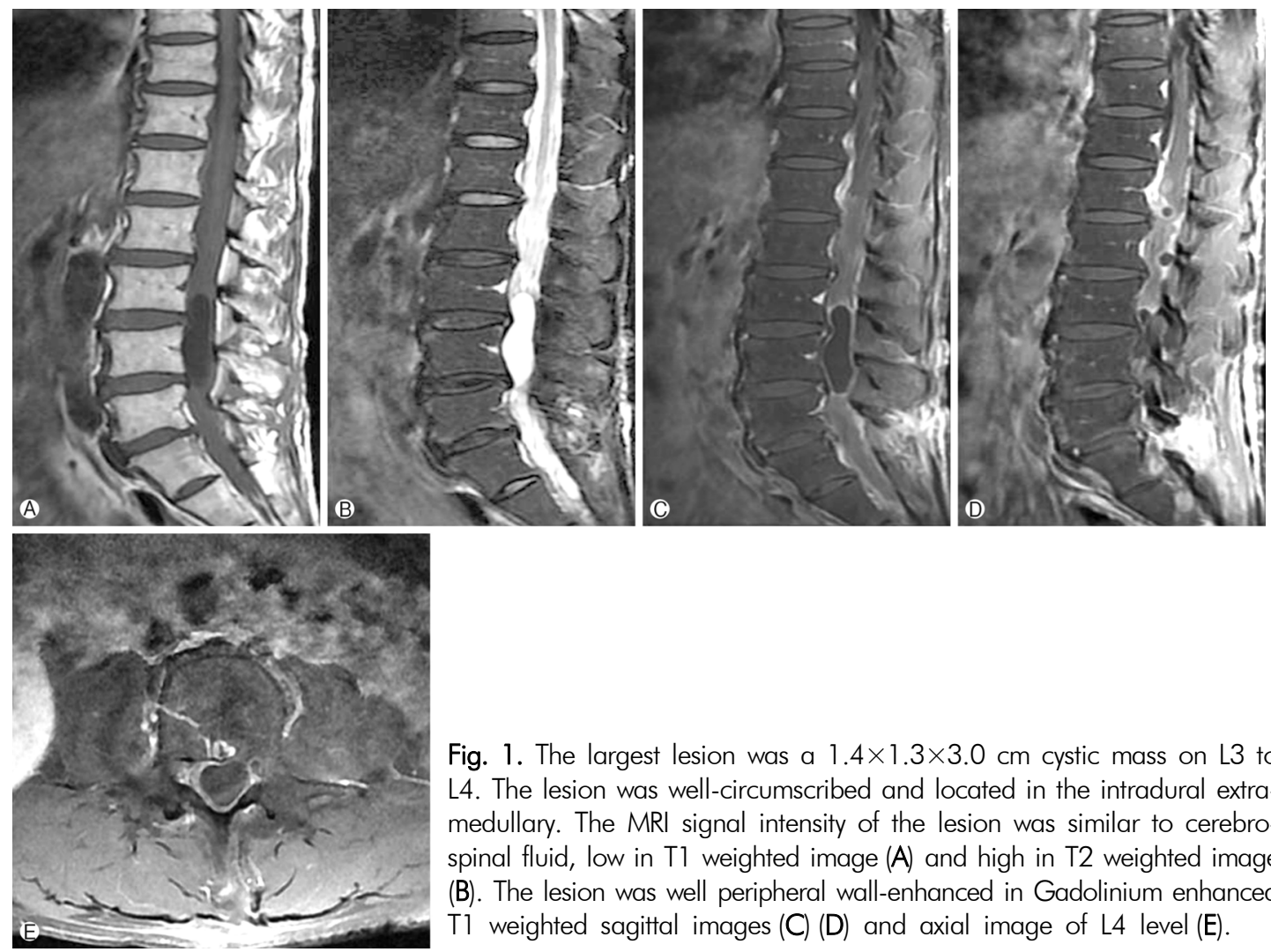

Fig. 1. The largest lesion was a $1.4 \times 1.3 \times 3.0 \mathrm{~cm}$ cystic mass on $\mathrm{L} 3$ to L4. The lesion was well-circumscribed and located in the intradural extramedullary. The MRI signal intensity of the lesion was similar to cerebrospinal fluid, low in $\mathrm{T} 1$ weighted image (A) and high in T2 weighted image (B). The lesion was well peripheral wall-enhanced in Gadolinium enhanced T1 weighted sagittal images (C) (D) and axial image of $L 4$ level (E).

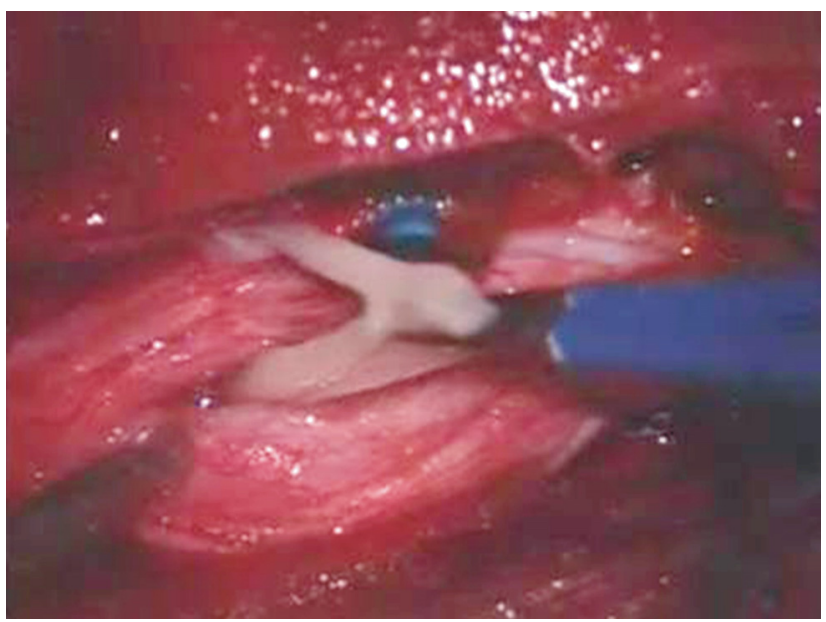

Fig. 2. Intraoperative photograph showing whitish and mucoid cysticerci.

adhesions between the dura and spinal nerve roots. Dissection proceeded, revealing abnormal cystic masses compressing the nerve roots. The cyst was punctured, spilling clear mucoid fluid into the surgical field. The exposed cysticerci, white and mucoid, was easily removed (Fig. 2). After internal decompression, the spinal nerve roots swelled. The dura was closed watertightly. No significant change was observed with nerve

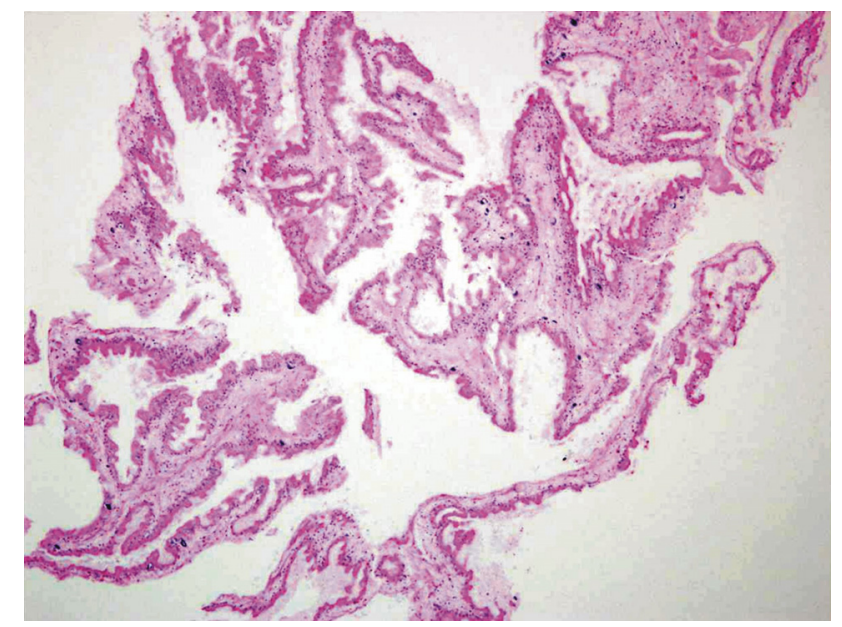

Fig. 3. A microphotograph indicates an eosinophilic outer cuticle layer and single layered subcuticle cell, representing typical cysticercosis findings (Hematoxylin and eosin stain $x 100$ ).

detectors after the surgery. Bilateral radiating pain in lower limbs were improved postoperatively, however, patient showed bilateral motor weakness (GIII) in both legs. Steroids were administered and the motor weakness improved daily. Four days later, the patient experienced symptomatic improvement without further neurologic deficits except for mild sensory impairments. After the operation he was treated with 
albendazole ( $15 \mathrm{mg} / \mathrm{kg} /$ day), and no additional complications were observed. The pathologic findings showed an eosinophilic outer cuticle layer and single layered subcuticle cell, representing typical cysticercosis findings (Fig. 3). MRI, taken 2 months later, showed removal of the largest cystic mass and removal or reduction of the small cystic masses observed from L1 to L5.

\section{DISCUSSION}

Cysticercosis, the most common parasitic disease, caused when Taenia Solium invades the central nervous system ${ }^{1)}$. The pig is the intermediate host and human is generally the definite host, although sometimes the human becomes the intermediate host by chance ${ }^{1,5,6}$. CNS involvement of the neurocysticerosis is mostly in the brain and spinal involvement is very rare ${ }^{15}$. Most authors showed an incidence of spinal neurocysticercosis to be of $1.5-3 \%$ of all cases ${ }^{1,2,15)}$. In addition, spinal neurocysticerosis without brain involvement is even rarer ${ }^{1,9,13)}$.

The egg of the Taenia Solium parasite reaches the intestinal system when consumed. The pancreatic enzyme then digests the eggshell and releases the embryo, known as the oncosphere. The embryo invades the intestinal wall and migrates through the blood stream and develops to the larval stage ${ }^{5)}$. Spinal neurocysticercosis is classified as intradural form and extradural form, depending on its location. The latter is very rare. The intradural form consists of a subarachnoid lesion and an intramedullary lesion, and the former is more common. It is thought that subarachnoid cysticercosis is caused by direct CSF dissemination, and intramedullary cysticercosis is caused by hematogenous dissemination ${ }^{4,8,9)}$.

The signs and symptom of spinal neurocysticercosis may vary depending on the location and size of the lesion ${ }^{1)}$. One of the important pathophysiological mechanisms is the direct mass effect, possibly causing muscle strength deterioration from myelopathy due to cord compression ${ }^{2)}$. Inflammatory reaction may also cause symptoms caused mainly by the parasite metabolism or by cyst degeneration. The mechanisms which may cause other symptoms include meningitis or cord degeneration caused by vascular insufficiency ${ }^{1,10)}$.

In serologic diagnosis, serum and CSF ELISA tests helped diagnose cysticercosis ${ }^{3,6,12}$. The sensitivity and the specificity of the ELISA test are about $50 \%$ and $70 \%$, for serum and about $87 \%$ and $97 \%$ for CSF, respectively ${ }^{12}$.

MRI is the best method to diagnose spinal neurocysticercosis. Rahalkar et al. categorized MRI findings in four stages ${ }^{11}$. First is the vesicular stage where the parasite lives. The cystic lesion is shown to be hypointense in $\mathrm{T} 1$ but hyperintense in $\mathrm{T} 2$ without considering the surrounding edema. Second is the colloidal vesicular stage, and the edema shows a lesion by an immune response when the parasite dies. The cystic lesion appears as a ring enhancing lesion in contrast enhancement MRI due to the defect in BBB. Third is the granulonodular stage where the capsule thickens and calcification begins. The last is the calcified nodular stage when the parasite is already dead. A calcified lesion is difficult to observe in the MRI, and CT imaging can be better in lesion observation.

There are various methods to diagnose spinal neurocysticercosis, but only surgical section and the pathological examinations provide a definite diagnosis ${ }^{6}$.

The treatment for spinal neurocysticercosis consists of medicine and surgery. The treatment of choice for the former includes anticysticercal drugs, like albendazole and praziquantel $^{3,5,6}$. Albendazole is preferred over praziquantel by many researchers due to a higher CSF penetration of the drug and a possible drug serum concentration increase in combined administration of steroids ${ }^{6,14)}$. Steroids shall be administered with anticysticercal drugs because acute neurological degradation may occur with inflammatory reactions following the parasite death ${ }^{3)}$. Also, the threshold for symptom aggravation in the spinal canal is very narrow compared to that of other organs due to its structural characteristics. Therefore, surgical decompression shall be immediately performed in the case of neurological symptom aggravation during the treatment of spinal neurocysticercosis ${ }^{1)}$.

The indication for surgical treatment of spinal neurocysticercosis includes very severe symptoms, symptom aggravation, medical treatment failure, and acute aggravation of neurological symptoms during treatment ${ }^{1)}$. The purpose of the surgery is to excise the lesion as much as possible while avoiding neurological aggravation. Sometimes, cystic lesions may show severe adhesion to the surrounding tissues due to arachnoiditis. The CSF flow may be blocked by this severe adhesion of the arachnoiditis, and treatment that reforms CSF may be required by duroplasty or shunt ${ }^{1)}$. Cystic migration may occur, and the lesion found in the MRI or CT image may be different from the lesion during surgery ${ }^{7)}$. Therefore, it should be considered to perform an imaging again if the time period between the MRI or CT and the surgery is long.

\section{CONCLUSION}

Spinal neurocysiticercosis is rare and isolated spinal neurocysticerosis is even rarer. However, all should be considered in differential diagnosis of a cystic lesion occupying the spinal space. Spinal neurocysiticercosis may be satisfactorily managed with surgery and medication. 


\section{REFERENCES}

1. Alsina GA, Johnson JP, McBride DQ, Rhoten PR, Mehringer $\mathrm{CM}$, Stokes JK: Spinal neurocysticercosis. Neurosurg Focus 12: e8, 2002

2. Canelas HM, Ricciardi-Cruz O, Escalante AD: Cysticercosis of the Nervous System: Less Frequent Clinical Forms. Iii. Spinal Cord Forms. Arq Neuropsiquiatr 21:77-86, 1963

3. Corral I, Quereda C, Moreno A, Lopez-Velez R, Martinez-SanMillan J, Guerrero A, et al: Intramedullary cysticercosis cured with drug treatment. A case report. Spine (Phila Pa 1976) 21: 2284-2287, 1996

4. De Souza Queiroz L, Filho AP, Callegaro D, De Faria LL: Intramedullary cysticercosis. Case report, literature review and comments on pathogenesis. J Neurol Sci 26:61-70, 1975

5. Hawk MW, Shahlaie K, Kim KD, Theis JH: Neurocysticercosis: a review. Surg Neurol 63:123-132; discussion 132, 2005

6. Homans J, Khoo L, Chen T, Commins DL, Ahmed J, Kovacs A: Spinal intramedullary cysticercosis in a five-year-old child: case report and review of the literature. Pediatr Infect Dis J 20:904-908, 2001

7. Kim KS, Weinberg PE: Spinal cysticercosis. Surg Neurol 24: 80-82, 1985
8. Kishore LT, Gayatri K, Naidu MR, Mateen MA, Dinakar I, Ratnakar KS: Intermedullary spinal cord cysticercosis--a case report and literature review. Indian J Pathol Microbiol 34:219221, 1991

9. Leite CC, Jinkins JR, Escobar BE, Magalhaes AC, Gomes GC, Dib G, et al: MR imaging of intramedullary and intraduralextramedullary spinal cysticercosis. AJR Am J Roentgenol 169: 1713-1717, 1997

10. Proano JV, Madrazo I, Garcia L, Garcia-Torres E, Correa D: Albendazole and praziquantel treatment in neurocysticercosis of the fourth ventricle. J Neurosurg 87:29-33, 1997

11. Rahalkar MD, Shetty DD, Kelkar AB, Kelkar AA, Kinare AS, Ambardekar ST: The many faces of cysticercosis. Clin Radiol 55:668-674, 2000

12. Rosas N, Sotelo J, Nieto D: ELISA in the diagnosis of neurocysticercosis. Arch Neurol 43:353-356, 1986

13. Sheehan JP, Sheehan JM, Lopes MB, Jane JA: Intramedullary cervical spine cysticercosis. Acta Neurochir (Wien) 144:10611063, 2002

14. Sotelo J, Del Brutto OH: Review of neurocysticercosis. Neurosurg Focus 12:e1, 2002

15. Sotelo J, Guerrero V, Rubio F: Neurocysticercosis: a new classification based on active and inactive forms. A study of 753 cases. Arch Intern Med 145:442-445, 1985 